\title{
Failure Prediction of High-Capacity Electrode Materials in Lithium-Ion Batteries
}

\author{
Chengpeng Wang, ${ }^{a}$ Zengsheng Ma, ${ }^{a, z}$ Yan Wang, ${ }^{b}$ and Chunsheng $\mathrm{Lu}^{\mathrm{c}, \mathrm{z}}$ \\ ${ }^{a}$ National-Provincial Laboratory of Special Function Thin Film Materials, and School of Materials Science and \\ Engineering, Xiangtan University, Hunan 411105, People's Republic of China \\ ${ }^{b}$ School of Information and Electronic Engineering, Hunan University of Science and Technology, Hunan 411201, \\ People's Republic of China \\ ${ }^{c}$ Department of Mechanical Engineering, Curtin University, Perth, WA 6845, Australia
}

The large volume change during lithium-ion insertion/extraction leads to huge stress and even failure of active materials. To well understand such a problem, the two-phase lithiation process of film and hollow core-shell electrodes is simulated by using a non-linear diffusion lithiation model. The dynamic evolution of lithium-ion concentration and diffusion-induced stress are obtained. Based on the dimensional analysis, a phase diagram is determined to demonstrate the relationship between critical failure, structure dimensions and mechanical properties. As a case study, the critical state of charge in Sn films are measured and compared with theoretical results. (C) The Author(s) 2016. Published by ECS. This is an open access article distributed under the terms of the Creative Commons Attribution Non-Commercial No Derivatives 4.0 License (CC BY-NC-ND, http://creativecommons.org/licenses/by-nc-nd/4.0/), which permits non-commercial reuse, distribution, and reproduction in any medium, provided the original work is not changed in any way and is properly cited. For permission for commercial reuse, please email: oa@electrochem.org. [DOI: 10.1149/2.0251607jes] All rights reserved.

Manuscript submitted December 31, 2015; revised manuscript received March 3, 2016. Published April 5, 2016.

Because of the large storage capacity and high energy density, lithium-ion batteries (LIBs), one of the most promising secondary cells, ${ }^{1-3}$ have been widely used in portable electronic devices. Recently, more research interest has focused on their potential applications in electric vehicles., ${ }^{2,45}$ As typical high-capacity electrode materials (e.g., Si, Ge, Sn, and some transition metal oxides) can host a large amount of $\mathrm{Li}$-ions, this makes them promising candidates for demanding applications. ${ }^{6,7}$ For instance, Si has the highest theoretical specific capacity in the phase of $\mathrm{Li}_{22} \mathrm{Si}_{5}$ (up to $4200 \mathrm{~mA} \mathrm{~h} \mathrm{~g}$ ), which is nearly ten times higher than that of fully-lithiated graphite in $\mathrm{LiC}_{6}$ (372 $\left.\mathrm{mA} \mathrm{h} \mathrm{g}^{-1}\right){ }^{8,9}$ Other electrode materials such as Ge and $\mathrm{Sn}$ also have considerable theoretical specific capacities $\left(1623 \mathrm{~mA} \mathrm{~h} \mathrm{~g}^{-1}\right.$ for $\mathrm{Li}_{22} \mathrm{Ge}_{5}$ and $700 \mathrm{~mA} \mathrm{~h} \mathrm{~g}{ }^{-1}$ for $\left.\mathrm{Li}_{22} \mathrm{Sn}_{5}\right) .4,7,10$

However, the large number of Li-ions inserting into high-capacity electrode materials may result in a huge volume change $(400 \%$ for full lithiation of $\mathrm{Si}){ }^{6}$ and a series of shortcomings: fracture or pulverization of active materials, breakage of a conduction path for electrons and lose of electrical contact, and destruction of solid electrolyte interphase formed by the reaction between active materials and electrolyte. They can rapidly fade electrochemical properties of active materials and result persistent decrease of their long-term coulombic efficiency. ${ }^{11-15}$

To solve these problems, extensive efforts have been made over the last decade. It is shown that nanostructure-based battery electrodes such as nanofilms, nanowires and nanoparticles, can alleviate diffusion-induced stress and improve their cycle life through structural optimization and geometric restriction. ${ }^{16-20}$ For example, a facile and scalable in situ chemical vapor deposition technique was developed for one-step fabrication of three-dimensional porous networks anchored with $\mathrm{Sn}$ nanoparticles $(5-30 \mathrm{~nm})$ and encapsulated with graphene shells of about $1 \mathrm{~nm}$ as a superior LIB anode. ${ }^{21}$ However, the irreversible capacity loss caused by stress damage during the first cycle is still serious. ${ }^{6,22,23}$ Due to complicated lithiation deformation mechanisms, ${ }^{11,24}$ the study on the structural change and stress evolution of high-capacity electrode materials during charging and discharging is necessary for the control of a large volume change and optimization of electrode structures.

Golmon et al. ${ }^{25}$ investigated the evolution of concentration, displacement, and stress in $\mathrm{Si}$ particles during the first insertion of $\mathrm{Li}$-ion and found that diffusion-induced stress may be beyond the critical failure stress that depends on the particle size and discharge rate. Zhao et al. ${ }^{11}$ studied fracture and debonding in LIBs with hollow core-shell

żE-mail: zsma@xtu.edu.cn; c.lu@ curtin.edu.au nano-structural electrodes and identified the conditions to avert fracture and debonding in terms of the core radius, shell thickness, and state of charge (SOC). Recent experiments by in situ transmission electron microscopy have revealed that there is a sharp phase boundary separated the lithiated amorphous $\mathrm{Li}_{x} \mathrm{Si}(x=3.75)$ phase from unlithiated crystalline Si phase, and during lithiation, crack initiation on the surface of a sphere..$^{5,26-28}$ The two-phase lithiation mechanism is different from a previous understanding of what $\mathrm{Li}$-poor and $\mathrm{Li}$ rich phases transform continuously into each other with changing composition. ${ }^{9,29,30}$ Conversely, there is a large solubility gap between these two phases, manifesting an abrupt change in Li-ion concentrations across the phase boundary. ${ }^{5,31}$ On the basis of kinetics measurements, this electrochemical behavior is believed as the rate-limiting effect of chemical bond breaking. ${ }^{32,33}$ Liu et al. ${ }^{26}$ established an elastoplastic lithiation model, in which the effective diffusion coefficient was assumed non-linearly dependent on the local Li-ion concentration. The unexpected surface cracking is attributed to the development of hoop tension in the surface layer. It is diametrically opposite to results inferred from single-phase modeling, which predicted only compressive hoop stress in the surface layer and thus crack initiation from the center of lithiated particles and wires. ${ }^{9,29,34}$ Huang et al. ${ }^{31}$ obtained the stress distribution by a finite difference method and presented a transparent mechanical framework, integrating multiphase microstructure and large volume change.

Although a lot of theoretical models and experiments have been done on diffusion-induced stress and structural failure in high-capacity electrode materials, there is still lack of a good understanding on the detailed dynamic evolution of stress field induced by large volume deformation, and the effects of structural size and material properties on electrochemical performance. In this paper, we aim to find out the relationships between material shape factors, mechanical properties and electrochemistry under different SOCs during Li-ion inserting in electrodes.

\section{Model and Numerical Analysis}

Diffusion equations of two-phase lithiation.-The transport of Li-ions in an electrode can be modeled as a concentration-driven bulk diffusion process, and the diffusion flux of Li-ions, $\boldsymbol{J}$, can be represented as

$$
\boldsymbol{J}=-D \nabla C
$$

where $D$ is the effective diffusion coefficient depending on concentration, $C$ is the molar concentration of Li-ions, and $\nabla$ is the gradient 
operator. The governing equation for mass conservation is

$$
\int_{V} \frac{d C}{d t} d V+\int_{S} \boldsymbol{n} \cdot \boldsymbol{J} d S=0
$$

where $t$ is the lithiated time, $V$ is the volume with a surface area $S$, and $\boldsymbol{n} \cdot \boldsymbol{J}$ is the flux of concentration with $\boldsymbol{n}$ the outward normal to the surface. Here, it is worth noting that the equation for mass conservation in diffusion is analogous to one for energy balance in heat transfer provided that the product of density and specific heat terms is equal to 1 .

Zhang et al. ${ }^{35}$ systematically studied the intercalation-induced stress developed in $\mathrm{LiMn}_{2} \mathrm{O}_{4}$ particles with various shapes and sizes, and based on a diffusion-stress coupling model, they showed that the concentration profile has a smaller gradient than that without the stress effect. However, recent experiments have exhibited that there is a sharp phase boundary between lithiated and unlithiated phases in a partially lithiated high-capacity electrode material. ${ }^{5,27}$ That is, the Li-ion concentration changes abruptly in the reaction front during lithiation. In order to obtain such a two-phase lithiation process, a non-linear diffusion model was applied, describing a sharply phase transition of diffusion rate at high $\mathrm{Li}$-ion concentrations. ${ }^{26,36}$ It is assumed that the effective diffusion coefficient $D$ is non-linearly dependent on the local Li-ion concentration $C$, that is

$$
D=D_{0} C \frac{\mathrm{d}^{2} f}{\mathrm{~d} C^{2}}
$$

where $D_{0}$ is the diffusion constant and $f$ is the free energy. On the basis of a regulation solution model, the free energy function can be represented as

$$
f=\Phi C(1-C)+[C \ln C+(1-C) \ln (1-C)]
$$

Thus, Eq. 3 can be deduced as

$$
D=D_{0}\left[\frac{1}{1-C}-2 \Phi C\right]
$$

where $\Phi$ is a tuned constant to control the concentration profile near the reaction front. The $\mathrm{Li}$-ion concentration $C$ is governed by the standard diffusion equation and normalized by the maximum Li-ion concentration $\left(C_{0}\right)$ at the fully lithiated state. In diffusion simulations, the normalized $\mathrm{Li}$-ion concentration $\left(C / C_{0}\right)$ behind the reaction interface can quickly reach a high value of about 1 , while that ahead of the front remains nearly zero. This produces a sharp reaction front, ${ }^{37}$ which provides a basis for further analysis on stress.

Elastic-perfectly plastic model of lithiation.-To understand the mechanism of mechanical failure, a stress model for a high power lithium-ion battery was developed by Fu et al. ${ }^{38}$ based on an electrochemical and thermal model, where electrodes are regarded as elastic materials whose expansion is affected by concentration and partial molar volume of Li-ions. In contrast, the present model accounts for the plastic deformation that may readily occur during lithiation of high-capacity materials. ${ }^{26,39}$ Therefore, we adopt an elastic-perfectly plastic model to evaluate lithiation-induced deformation and stress states. The increment of the total strain, $d \varepsilon_{i j}$, can be written as the sum of three contributions, that is

$$
d \varepsilon_{i j}=d \varepsilon_{i j}^{c}+d \varepsilon_{i j}^{e}+d \varepsilon_{i j}^{p}
$$

where the increment of lithiation-induced chemical strain, $d \varepsilon_{i j}^{c}$, is proportional to that of $\mathrm{Li}$-ion concentration, or

$$
d \varepsilon_{i j}^{c}=\beta d c
$$

with $\beta=\Omega \delta_{i j} / 3$ representing the expansion coefficient. Here, $\Omega$ is the partial molar volume of solute, and $\delta_{i j}=1$ for $i=j$, and otherwise, $\delta_{i j}=0$. The increment of elastic strain, $d \varepsilon_{i j}^{e}$, obeys Hooke's law

$$
d \varepsilon_{i j}^{e}=\left[(1+\nu) d \sigma_{i j}-\nu d \sigma_{k k} \delta_{i j}\right] / E
$$

where $E$ is Young's modulus, $v$ is Poisson's ratio, and repeated indexes mean summation. The increment of plastic strain, $d \varepsilon_{i j}^{p}$, abides the classic $J_{2}$-flow rule, namely, plastic yielding occurs when the von Mises effective stress, $\sigma_{\text {eq }}=\sqrt{3 s_{i j} s_{i j} / 2}$, equals the yield strength $\sigma_{Y}$. Here $s_{i j}=\sigma_{i j}-\sigma_{k k} \delta_{i j} / 3$ is the deviatoric stress, and $d \varepsilon_{i j}^{p}$ is proportional to $s_{i j}$, given by

$$
d \varepsilon_{i j}^{p}=d \lambda s_{i j}
$$

where $\lambda$ is a scalar coefficient that can be determined by solving the boundary value problem.

Finite element analysis.-The two-phase diffusion and constitutive equations of elastic-plastic deformation are numerically solved by using the commercial finite element software package ABAQUS. In order to describe the evolution of diffusion and associated stress generation, fully coupled thermal diffusion-stress analysis is performed in direct finite element simulations. ${ }^{30}$

The electrode material is taken to be a homogeneous and isotropic elastic-perfectly plastic solid under plane-strain and quasi-static deformation. Transient analysis, where diffusion equations are integrated with a backward-difference scheme and the computed system is solved with Newton's method, is carried out in ABAQUS/Standard. A uniform quadrilateral sweeping mesh is used with first-order elements because the problem is highly non-linear. Fine meshing is applied in the region of stress concentration, and the element size is set to be $0.1 \%$ of the global dimension with the axisymmetric condition. Here, the diffusion properties are assigned to generate a stable two-phase structure. We take $\Phi=1.95$ and assign a constant $C_{0}$ on the surface. For numerical stability, the maximum $D$ is limited to $10^{4} \times D_{0} \cdot{ }^{31}$

\section{Results and Discussion}

Evolution of concentration and stress.-In the case of a film structure, substrate is assumed to be rigid due to its high stiffness. The film with an initial thickness of $h_{0}$ is bonded on substrate. The film surfaces are stress-free and the Li-ion concentration in it is zero initially. During lithiation, the film is surrounded by an invariant Liion concentration and subjected to a constant lithium flux $J_{0}$ on the top surface. Fig. 1a shows the contour of Li-ion concentration in a partially lithiated film at various times, where the red region represents full lithiation and the blue region means poor lithiation. Obviously, a two-phase lithiation process is obtained and there is a sharp phase boundary between lithiated and unlithiated materials. A significant expansion is caused by insertion of Li-ions, which agrees well with lithiation experiments. ${ }^{17}$ As shown in Fig. 1b, the Li-ion concentration is close to zero in the interior of film and approaches to 1 on its surface. The phase boundary at the mutation of concentration moves forward to the bottom of film with the process of lithiation. It is seen from Figs. $1 \mathrm{c}$ and $1 \mathrm{~d}$ that, in the early stage of lithiation, $\sigma_{x}$ is compressive in the surface layer of film. As lithiation proceeds to the late stage, $\sigma_{x}$ shifts from compression to tension, and reaches to the yield stress that provides the inducement of surface cracking. Similarly, the stress $\sigma_{y}$ reverses from compression to tension in the edge of bottom surface, indicating a significant influence on interfacial debonding of film electrode (see Fig. 1d). The stress exhibits a non-monotonic profile in a certain stage of lithiation process (e.g., at $120 \mathrm{~s}$ ), which may be due to two-way stress-diffusion coupling and interactive effect of diffusion-induced stress. ${ }^{35,40}$

The recent design of electrodes involves a hollow core-shell nanostructure, which can enhance both mechanical and chemical stability of high-capacity electrodes. ${ }^{41}$ As shown in Fig. 2a, the initial inner radius of a hollow particle is $a$, and its outer radius is $b$. The particle is coated by a stiff shell with an outer radius $c$. The shell separates the electrolyte and electrode, and forces the core to swell into hollow space. Here, the shell is assumed to be $\mathrm{Al}_{2} \mathrm{O}_{3}$ coating with Young's modulus of $300 \mathrm{GPa}$, and it is stress-free except for the interface between the shell and inner active particle. During lithiation, the outward expand is slight because the shell is much stronger than the internal hollow active material. The particle is surrounded by an invariant Liion concentration and subjected to a constant lithium flux $J_{0}$ on both the outer and inner surfaces. As shown in Fig. 2a, Li-ions can transfer 
(a)

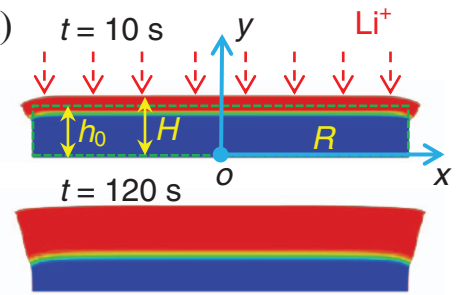

$t=600 \mathrm{~s}$
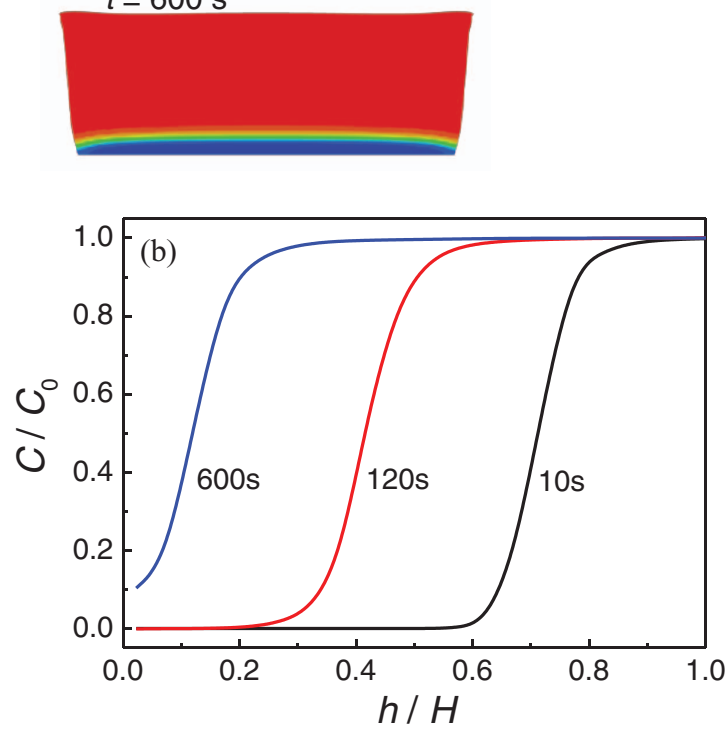
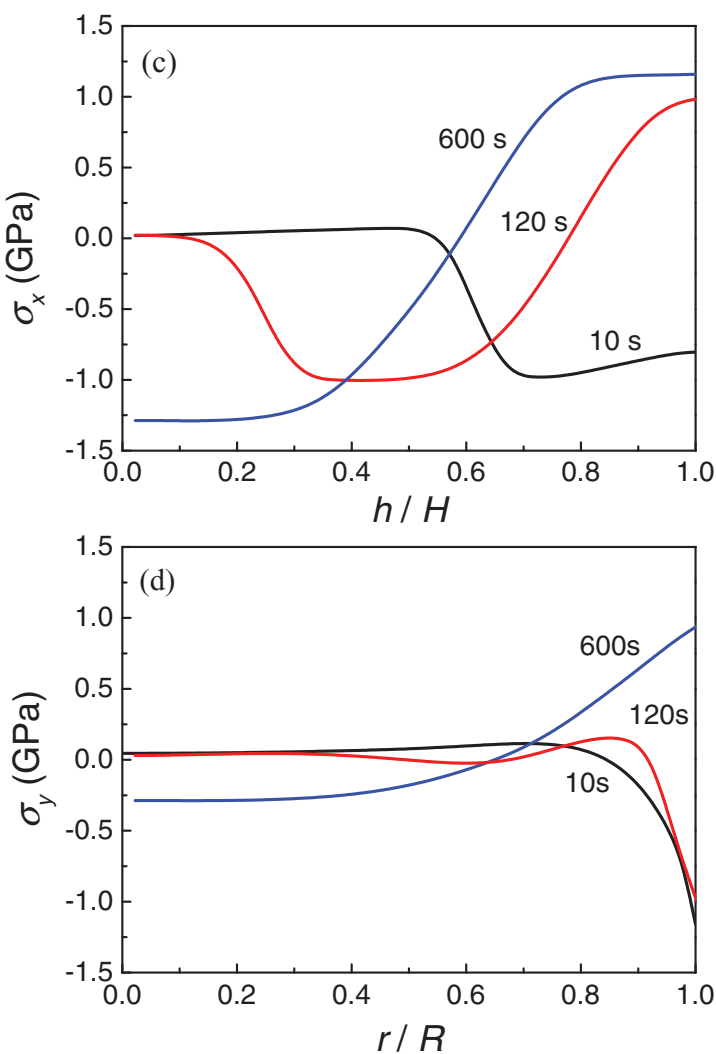

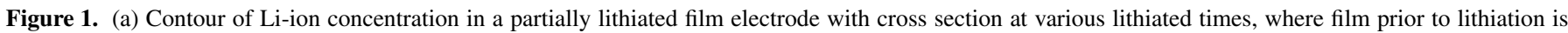

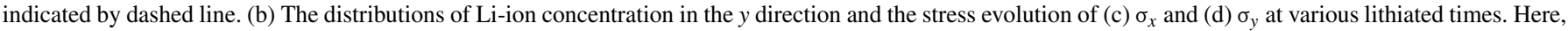
the vertical distance $h$ and horizontal distance $r$ are normalized by current thickness $H$ and radius $R$, respectively.

into active material through outer and inner surfaces, and thus a two sharp phase boundary appears in the hollow particle. As seen in Fig. $2 \mathrm{~b}, \mathrm{Li}$-ion concentration in the shell remains zero, and its distribution in active material is bilaterally symmetrical because of synchronous lithiation in both sides. With the process of lithiation, concentration changes abruptly in phase boundaries that migrate into the middle of active material. $\sigma_{\theta}$ is compressive in core due to constraint of the outer shell, and such an internal hoop compressive stress causes inward filling (see Fig. 2c). $\sigma_{\theta}$ transforms to a large tensile stress in the shell and continues to increase with lithiation proceeding, which may lead to shell fracture. This is consistent with previous studies by Zhao et al. ${ }^{11}$ Fig. 2 d exhibits the evolution of radial stress $\sigma_{r}$. The core-shell interface is firstly subjected to radial compression and then reverses to radial tension, which may result in core-shell debonding.

Failure modes.-Failure of electrode structures is mainly related to their dimensions and mechanical properties, and thus the critical conditions can be determined by interfacial fracture energies. In the case of a film structure, we have

$$
\Gamma=\Gamma\left(h_{0}, E, \sigma_{\mathrm{Y}}, \mathrm{SOC}\right)
$$

and similarly, in a hollow core-shell structure,

$$
\Gamma=\Gamma\left(c-b, b / a, E, \sigma_{\mathrm{Y}}, \mathrm{SOC}\right)
$$

where $\Gamma$ is the failure energy, $c-b$ is the thickness of a stiff shell, and $b / a$ represents the ratio of outer and inner radii of a hollow particle. Based on dimensional analysis, Eqs. 10 and 11 can be written by the non-dimensional functions as follows: for a film structure,

$$
\frac{\Gamma}{h_{0} E}=\Pi\left(\frac{\sigma_{\mathrm{Y}}}{E}, \mathrm{SOC}\right)
$$

and for a hollow core-shell structure,

$$
\frac{\Gamma}{(c-b) E}=\Pi\left(\frac{b}{a}, \frac{\sigma_{\mathrm{Y}}}{E}, \mathrm{SOC}\right)
$$

To completely fill the hollow space, Ma et al. ${ }^{24}$ demonstrated that the $b / a$ value is approximately equal to 1.1. Material parameters used in the models are listed in Table I. Based on simulations, the specific expression of these non-dimensional functions can be determined.

As shown in Fig. 3, at the beginning of lithiation (with a low SOC), film is under compression on surface and interface, and thus surface fracture or interfacial debonding does not occur. With continuous lithiation, surface and interfacial stresses transform from compression to tension and reach the critical value, leading to surface fracture and interfacial debonding. It is seen from Fig. 3a that there is a little influence of a relatively large $\sigma_{\mathrm{Y}} / E$ on the critical SOC. With the decrease of $\sigma_{\mathrm{Y}} / E$, the critical SOC increases rapidly and when $\sigma_{\mathrm{Y}} / E$ belows a certain value, there is no surface fracture even in full lithiation. For different initial thicknesses, although the tendency of curves is similar, the safe zone (area below fracture curves) increases with the decrease of $h_{0}$, indicating a better electrochemical property for a smaller thickness. Previous experiments also showed that a thinner film is favorable to delivery capacity and cycle stability. ${ }^{42}$ With the increase of $\sigma_{\mathrm{Y}} / E$ (see Fig. 3b), the critical SOC decreases continuously because of interfacial debonding. However, it is insensitive to SOC in the case of large $\sigma_{\mathrm{Y}} / E$ values, especially near 0.01 .

Based on the non-dimensional function of Eq. 12, the fitted specific expressions for a film structure are as follows: for surface fracture,

$$
\begin{aligned}
\mathrm{SOC}= & 0.12+\left\{45000 \cdot \exp \left[-7804\left(\sigma_{Y} / E\right)\right]+0.12\right\} \\
& \cdot\left\{\exp \left[4.66 \times 10^{6}\left(\Gamma / h_{0} E\right)\right]-1\right\}
\end{aligned}
$$



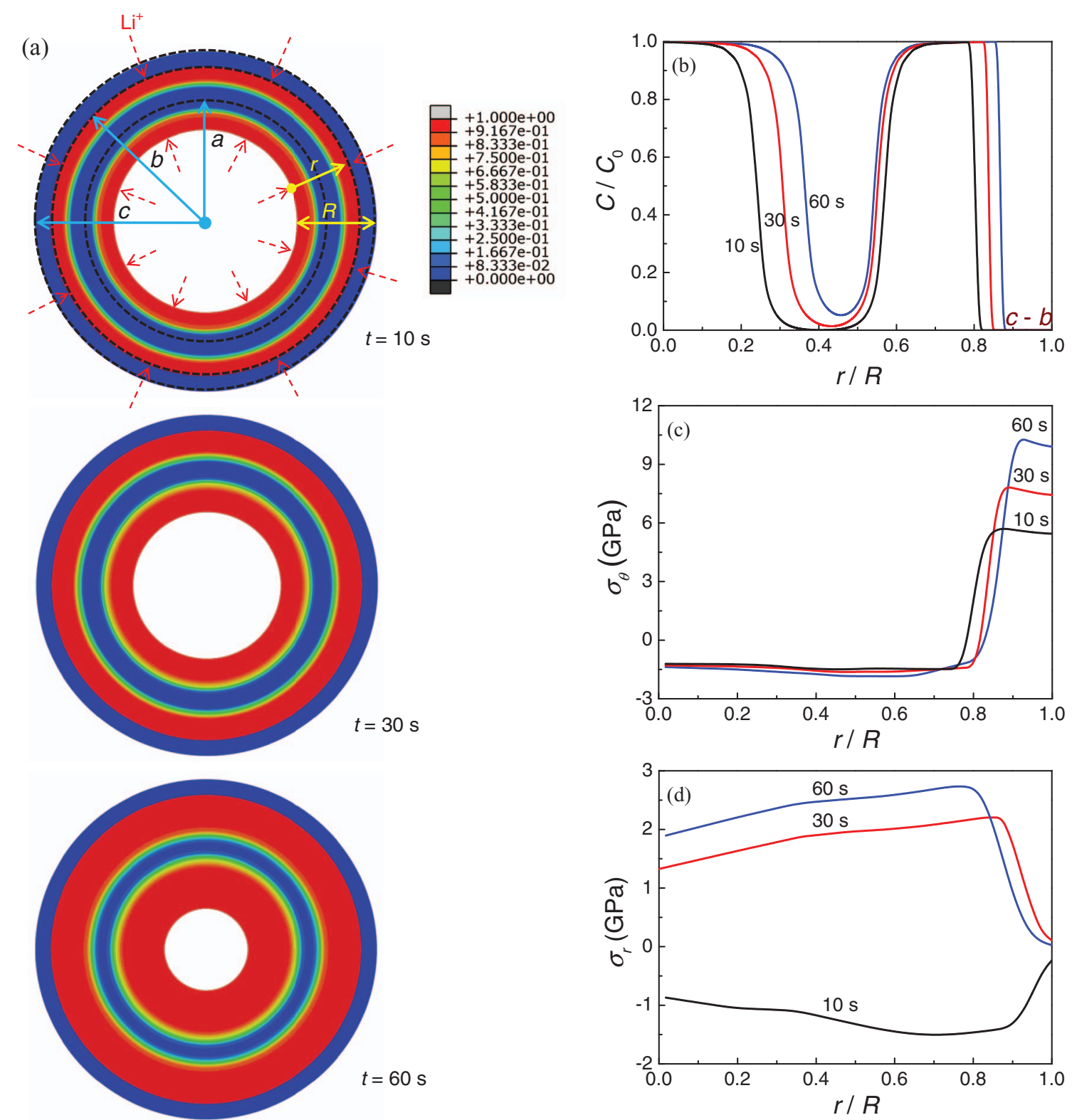

Figure 2. (a) Contour of Li-ion concentration in a partially lithiated spherical hollow core-shell electrode with cross section at various lithiated times, where particle prior to lithiation is indicated by dashed line. (b) Radial distributions of Li-ion concentration and the evolution of (c) hoop stress $\sigma_{\theta}$ and (d) radial stress $\sigma_{r}$ at various lithiated and delithiated times.

and for interfacial debonding,

$$
\mathrm{SOC}=\frac{1.67-84\left(\sigma_{Y} / E\right)+4.25 \times 10^{6}\left(\Gamma / h_{0} E\right)}{1+115\left(\sigma_{Y} / E\right)+1.25 \times 10^{7}\left(\Gamma / h_{0} E\right)}
$$

As shown in Fig. 4 a, it is safe for film electrodes with a smaller $\sigma_{\mathrm{Y}} / E$ and a bigger $\Gamma / h_{0} E$. Fracture does not happen even in full lithiation while $\sigma_{\mathrm{Y}} / E$ is small enough or $\Gamma / h_{0} E$ is sufficient large. However, the

\section{Table I. Material properties used in the models.}

\begin{tabular}{ccc} 
Parameter & Symbol & Value \\
\hline Failure energy & $\Gamma\left(\mathrm{pJ} / \mu \mathrm{m}^{2}\right)$ & $1-40$ \\
Young's modulus & $E(\mathrm{GPa})$ & $30-300$ \\
Poisson's ratio & $v$ & 0.22 \\
Yield strength & $\sigma_{\mathrm{Y}}(\mathrm{GPa})$ & $0.03-3$ \\
Film initial thickness & $h_{0}(\mu \mathrm{m})$ & $0.05-50$ \\
Stiff shell thickness & $c-b(\mathrm{~nm})$ & $5-50$
\end{tabular}

safe zone expands with the decrease of $\sigma_{\mathrm{Y}} / E$ and $\Gamma / h_{0} E$, and there is no debonding only if both of $\sigma_{\mathrm{Y}} / E$ and $\Gamma / h_{0} E$ are small enough (see Fig. 4b).

As shown in Fig. 5a, the critical SOC increases with the decrease of $\sigma_{\mathrm{Y}} / E$, and fracture does not occur even in full lithiation for a relatively small $\sigma_{\mathrm{Y}} / E$. The shell is more likely to fracture for a thinner shell. Debonding does not happen at the beginning of delithiation (with a high SOC) because of the radial compressive stress at interface (see Fig. 5b). With continuous delithiation, the radial stress converts from compression to tension and interfacial debonding initiates. The critical SOC also increases with the decrease of $\sigma_{\mathrm{Y}} / E$ and finally tends to a stable value with the increase of $c-b$. The safe zone (area above debonding curves) decreases with the increase of shell thickness.

Similarly, the fitted specific expressions for a hollow core-shell structure based on Eq. 13 are as follows: for shell fracture,

$$
\mathrm{SOC}=2.4+2.42 \times 10^{-2} /\left(\sigma_{\mathrm{Y}} / E\right)^{0.5}+23.77 / \log [\Gamma /(c-b) E]
$$



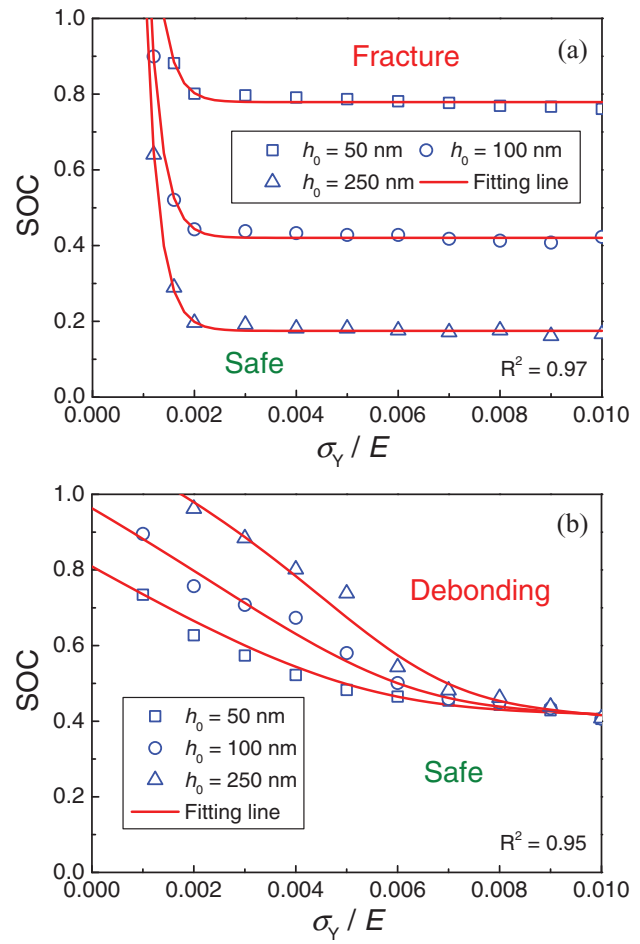

Figure 3. (a) Surface fracture and (b) interfacial debonding for the critical SOC with various $\sigma_{\mathrm{Y}} / E$. The discrete points and curves are finite element and fitting results, respectively.
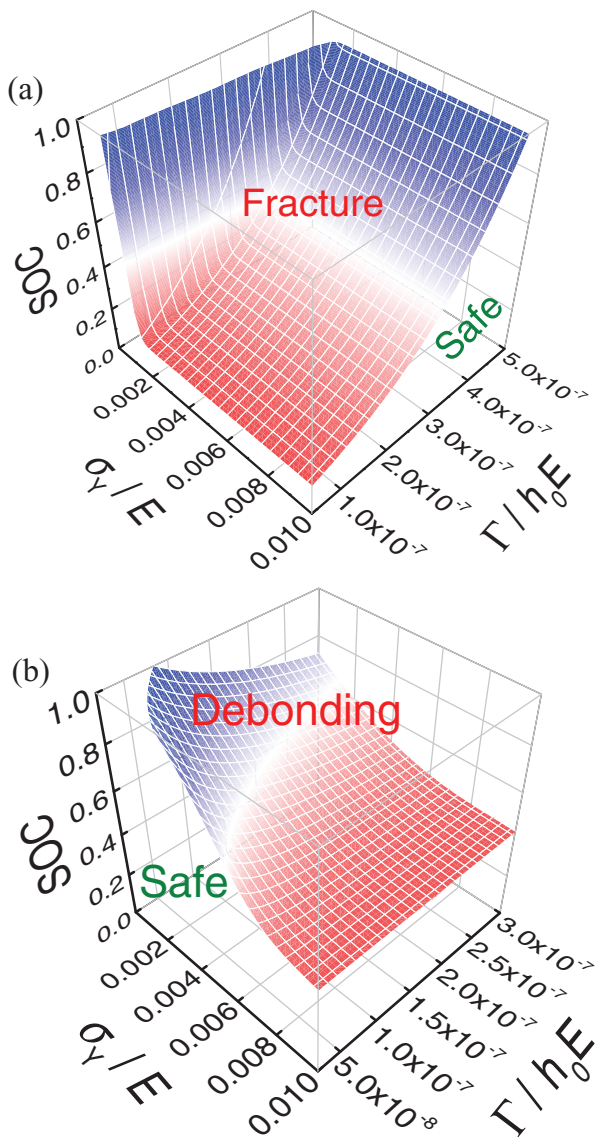

Figure 4. Failure mechanism diagrams based on the relationships between $\sigma_{\mathrm{Y}} / E, \Gamma / h_{0} E$ and critical SOC for film electrode: (a) surface fracture and (b) interfacial debonding.
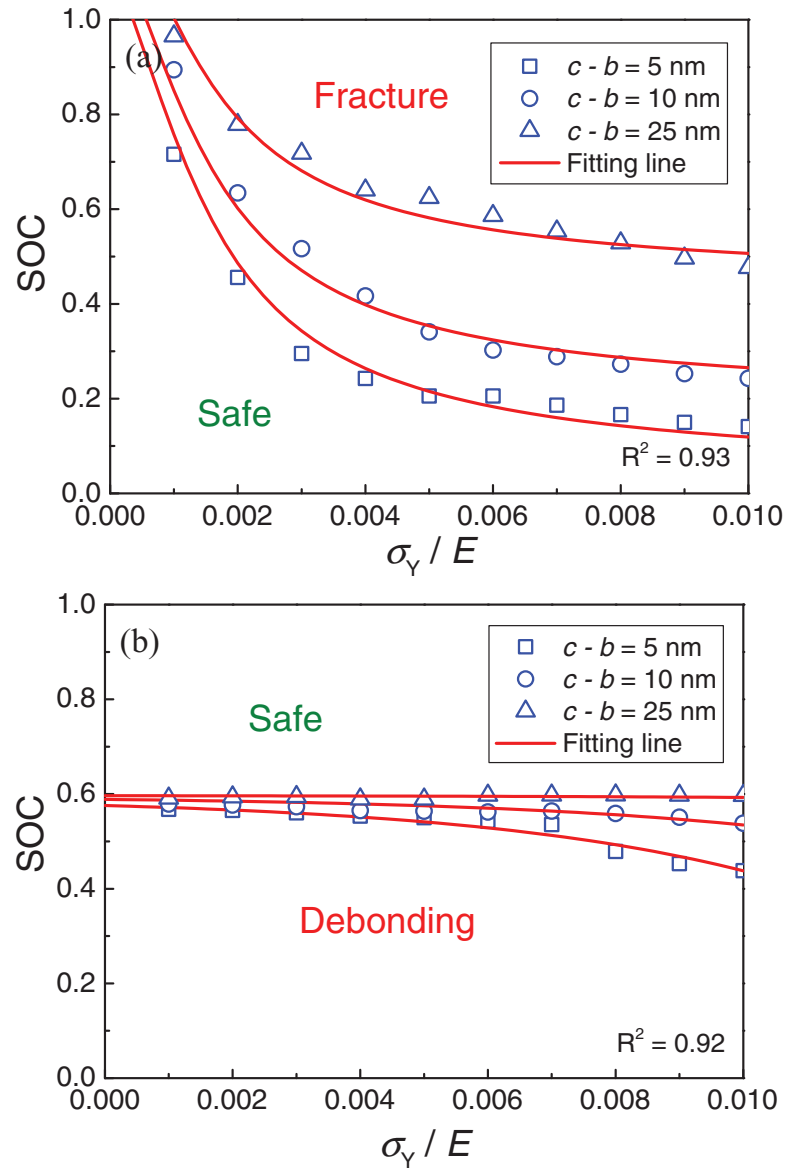

Figure 5. (a) Shell fracture and (b) interfacial debonding for the critical SOC with various $\sigma_{\mathrm{Y}} / E$ for a spherical hollow core-shell electrode. The discrete points and curves are finite element and fitting results, respectively.

and for interfacial debonding,

$$
\begin{aligned}
\mathrm{SOC}= & 0.6+\left\{81.14 \cdot \exp \left[220\left(\sigma_{\mathrm{Y}} / E\right)\right]\right. \\
& +18.65\} \cdot\{\exp [-130 \Gamma /(c-b) E]-1\}
\end{aligned}
$$

Accordingly, the corresponding three-dimensional phase diagrams of shell fracture and interfacial debonding are mapped in Fig. 6. It is seen from Fig. 6a that, the safe zone increases with the decrease of $\sigma_{\mathrm{Y}} / E$ and $\Gamma /(c-b) E$ during lithiation. While $\sigma_{\mathrm{Y}} / E$ is below a certain value, shell does not suffer fracture permanently. However, interface seems to be safe with the increase of $\sigma_{\mathrm{Y}} / E$ and $\Gamma /(c-b) E$ (see Fig. 6b), and there is no debonding even in full delithiation while both $\sigma_{\mathrm{Y}} / E$ and $\Gamma /(c-b) E$ are large enough.

Experimental verification.-To prove theoretical results, we prepared Sn film on smooth copper substrate by electroplating. Through controlling plating time, we obtained Sn films with different thicknesses, which were assembled into button cells (CR2016) as negative electrodes. Here, SOC can be determined by $\mathrm{SOC}=Q^{\prime} / Q$, with $Q^{\prime}$ and $Q$ being current and full lithiation capacities, respectively.

The surface topography of Sn film with initial thickness $h_{0}=10$ $\mu \mathrm{m}$ reveals a significant change with the charging process, as shown in Fig. 7. For SOC $=0.1$, the surface is smooth and flat. When SOC increases to 0.5 , the film roughness is grown slightly due to Li-ion insertion, and fluctuation extends obviously with SOC $=0.93$. Finally, a number of microcracks appear on surface at full lithiuation. Furthermore, with the increase of thickness, surface cracks occur easily for a lower SOC. For example, it is obvious that fracture begins when SOCs are 0.72 and 0.5 for initial thicknesses of $18 \mu \mathrm{m}$ and $26 \mu \mathrm{m}$, respectively. For film with initial thickness of $35 \mu \mathrm{m}$, as 


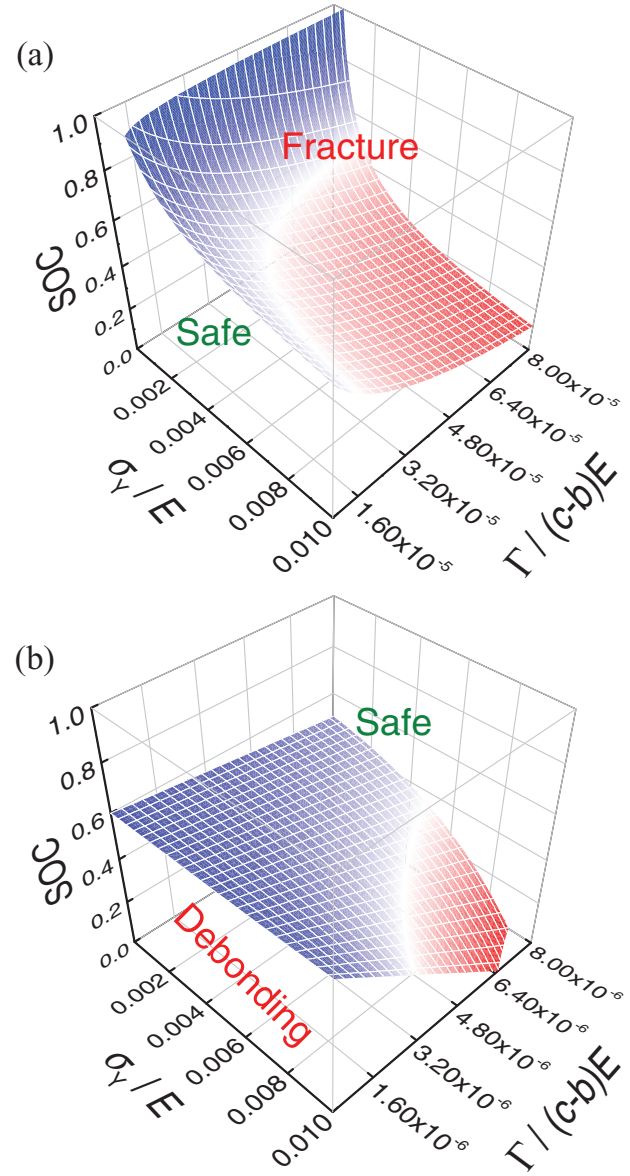

Figure 6. Failure mechanism diagrams based on the relationships between $\sigma_{\mathrm{Y}} / E, \Gamma /(c-b) E$ and critical SOC for a spherical hollow core-shell electrode: (a) shell fracture and (b) interfacial debonding.

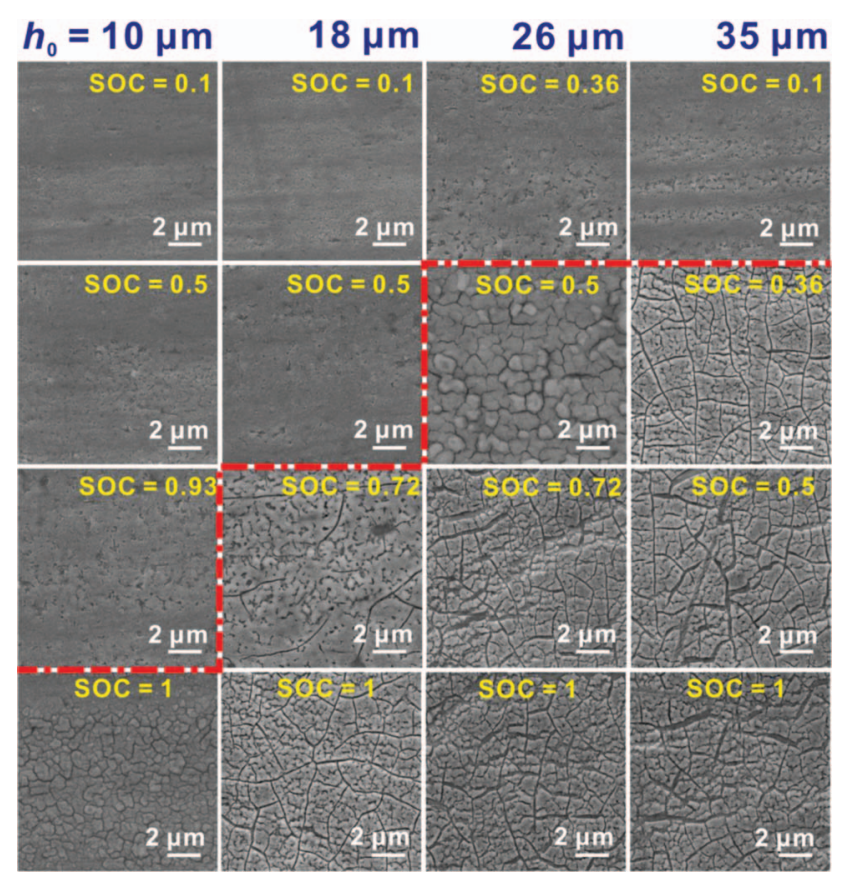

Figure 7. Surface topographies of Sn films with the initial thickness of $h_{0}=$ $10,18,26$, and $35 \mu \mathrm{m}$ under different SOCs.

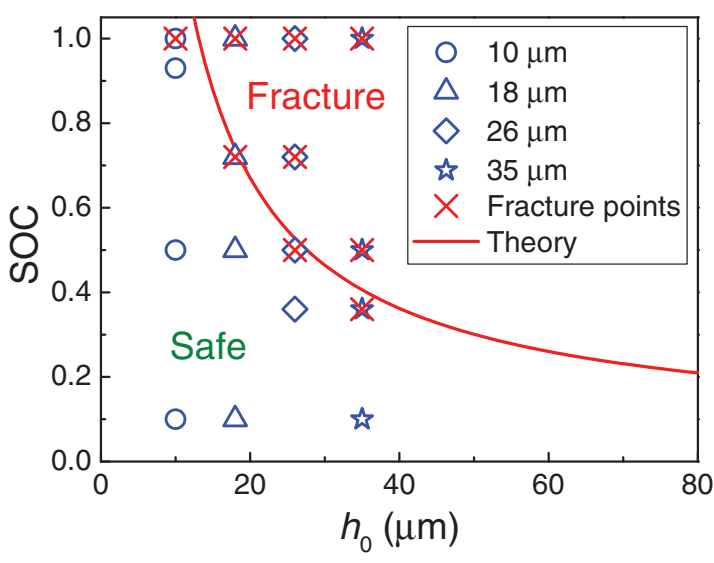

Figure 8. The critical condition of surface fracture for Sn films. The points and curve represent experimental values and theoretical results, respectively.

shown in Fig. 7, crack emerges while SOC reaches 0.36; and a large area of pulverization and peeling is observed as charging proceeds.

According to Eq. 14, the critical fracture curve of Sn film with different initial thicknesses can be plotted based on mechanical properties of Sn such as $\Gamma=5 \mathrm{~J} \mathrm{~m}^{-2}, E=40 \mathrm{GPa}$, and $\sigma_{\mathrm{Y}}=0.04 \mathrm{GPa}$ (see Fig. 8). It is shown that experimental values can be well described by theoretical results. The experimental critical SOC is slightly less than that of theoretical curves, which is mainly due to defects in actual materials.

\section{Conclusions}

Based on an elastic-perfectly plastic model and a finite element method, we have successfully established a relationship between the fracture energy, mechanical properties and critical SOC in a twophase lithiation process with the help of dimensional analysis. The relationship can be applied to predict surface fracture and interfacial debonding for film and hollow core-shell structural electrodes under different SOCs. Furthermore, we have demonstrated the accuracy of theoretical models by experimentally using Sn anodes deposited on copper substrate. The modeling highlights the strong fracture dependence on electrochemistry and stress in composite electrodes and provides important insight on the design of resilient LIBs.

\section{Acknowledgments}

This work was supported by the National Natural Science Foundation of China (grant nos. 11372267, 11402086 and 11472141).

\section{List of Symbols}

$a$

$b$

$c$

$C$

$C_{0}$

$D$

$D_{0}$

E

$E_{\mathrm{s}}$

$f$
$h$

$h_{0}$ initial inner radius of hollow particle, $\mathrm{nm}$ initial outer radius of hollow particle, $\mathrm{nm}$ initial outer radius of stiff shell, $\mathrm{nm}$ molar concentration of $\mathrm{Li}$-ions, $\mathrm{mol} / \mu \mathrm{m}^{3}$ maximum Li-ion concentration at fully lithiated state, $\mathrm{mol} / \mu \mathrm{m}^{3}$

effective diffusion coefficient, $\mu \mathrm{m}^{2} / \mathrm{s}$

diffusion constant, $\mu \mathrm{m}^{2} / \mathrm{s}$

Young's modulus, GPa

Young's modulus of stiff shell, GPa

free energy

vertical distance of film, $\mu \mathrm{m}$

initial thickness of film, $\mu \mathrm{m}$

thickness of film, $\mu \mathrm{m}$

diffusion flux, $\mathrm{mol} /\left(\mu \mathrm{m}^{2} \mathrm{~s}\right)$

lithium flux on electrode surface, $\mathrm{mol} /\left(\mu \mathrm{m}^{2} \mathrm{~s}\right)$

electrode surface normal 
$Q \quad$ fully lithiation capacity, $\mathrm{mAh} / \mathrm{g}$

$Q^{\prime} \quad$ current lithiation capacity, $\mathrm{mAh} / \mathrm{g}$

$r \quad$ horizontal or radial distance, $\mathrm{nm}$

$R \quad$ thickness of hollow core-shell electrode, $\mathrm{nm}$

$s_{i j} \quad$ deviatoric stress, GPa

$S \quad$ surface area, $\mu \mathrm{m}^{2}$

SOC state of charge

$t \quad$ lithiated time, $\mathrm{s}$

$V \quad$ volume, $\mu \mathrm{m}^{3}$

Greek

$\beta$

expansion coefficient of lithiation

total strain of lithiation

chemical strain of lithiation

elastic strain of lithiation

plastic strain of lithiation

concentration profile constant

failure energy, $\mathrm{pJ} / \mu \mathrm{m}^{2}$

scalar coefficient

Poisson's ratio

von Mises effective stress, GPa

yield strength, GPa

horizontal stress, GPa

vertical stress, GPa

hoop stress, GPa

radial stress, GPa

partial molar volume, $\mu \mathrm{m}^{3} / \mathrm{mol}$

\section{References}

1. J.-M. Tarascon and M. Armand, Nature, 414, 359 (2001).

2. K. T. Nam, D.-W. Kim, P. J. Yoo, C.-Y. Chiang, N. Meethong, P. T. Hammond, Y.-M. Chiang, and A. M. Belcher, Science, 312, 885 (2006).

3. B. Scrosati, J. Hassoun, and Y.-K. Sun, Energy Environ. Sci., 4, 3287 (2011).

4. N. Nitta, F. Wu, J. T. Lee, and G. Yushin, Mater. Today, 18, 252 (2015).

5. M. T. McDowell, S. W. Lee, J. T. Harris, B. A. Korgel, C. Wang, W. D. Nix, and Y. Cui, Nano Lett., 13, 758 (2013).

6. K. Zhao, M. Pharr, J. J. Vlassak, and Z. Suo, J. Appl. Phys., 108, 073517 (2010).

7. M. T. McDowell, S. W. Lee, W. D. Nix, and Y. Cui, Adv. Mater, 25, 4966 (2013)

8. J. W. Wang, X. H. Liu, K. Zhao, A. Palmer, E. Patten, D. Burton, S. X. Mao, Z. Suo, and J. Y. Huang, ACS Nano, 6, 9158 (2012).
9. Y Yao, M. T. McDowell, I. Ryu, H. Wu, N. Liu, L. Hu, W. D. Nix, and Y. Cui, Nano Lett., 11, 2949 (2011).

10. Y. Xu, Q. Liu, Y. Zhu, Y. Liu, A. Langrock, M. R. Zachariah, and C. Wang, Nano Lett., 13, 470 (2013).

11. K. Zhao, M. Pharr, L. Hartle, J. J. Vlassak, and Z. Suo, J. Power Sources, 218, 6 (2012).

12. S. C. Jung, J. W. Choi, and Y. K. Han, Nano Lett., 12, 5342 (2012).

13. A. Mukhopadhyay and B. W. Sheldon, Prog. Mater. Sci., 63, 58 (2014)

14. S. W. Lee, I. Ryu, W. D. Nix, and Y. Cui, Extreme Mech. Lett., 2, 15 (2015).

15. X. Gao, Z. Ma, W. Jiang, P. Zhang, Y. Wang, Y. Pan, and C. Lu, J. Power Sources, 311, 21 (2016).

16. Z. Ma, T. Li, Y. L. Huang, J. Liu, Y. Zhou, and D. Xue, RSC Adv., 3, 7398 (2013).

17. J. P. Maranchi, A. F. Hepp, A. G. Evans, N. T. Nuhfer, and P. N. Kumta, J. Electrochem. Soc., 153, A1246 (2006).

18. M. Liu, J. Power Sources, 275, 760 (2015).

19. Z. Jia and T. Li, J. Power Sources, 275, 866 (2015).

20. F. Hao and D. Fang, J. Electrochem. Soc., 160, A595 (2013).

21. J. Qin, C. He, N. Zhao, Z. Wang, C. Shi, E.-Z. Liu, and J. Li, ACS Nano, 8, 1728 (2014).

22. Z. Ma, Y. Zhou, J. Liu, D. Xue, Q. Yang, and Y. Pan, Adv. Mech., 43, 581 (2013).

23. R. Grantab and V. B. Shenoy, J. Electrochem. Soc., 159, A584 (2012).

24. Z. S. Ma, Z. C. Xie, Y. Wang, P. P. Zhang, Y. Pan, Y. C. Zhou, and C. Lu, J. Power Sources, 290, $114(2015)$

25. S. Golmon, K. Maute, S.-H. Lee, and M. L. Dunn, Appl. Phys. Lett., 97, 033111 (2010).

26. X. H. Liu, L. Zhong, S. Huang, S. X. Mao, T. Zhu, and J. Y. Huang, ACS Nano, 6 1522 (2012).

27. M. T. McDowell, S. W. Lee, C. Wang, W. D. Nix, and Y. Cui, Adv. Mater, 24, 6034 (2012).

28. S. C. Jung and Y.-K. Han, Electrochim. Acta, 62, 73 (2012).

29. I. Ryu, J. W. Choi, Y. Cui, and W. D. Nix, J. Mech. Phys. Solids, 59, 1717 (2011)

30. H. Haftbaradaran, J. Song, W. Curtin, and H. Gao, J. Power Sources, 196, 361 (2011).

31. S. Huang, F. Fan, J. Li, S. Zhang, and T. Zhu, Acta Mater., 61, 4354 (2013).

32. X. H. Liu, J. W. Wang, S. Huang, F. Fan, X. Huang, Y. Liu, S. Krylyuk, J. Yoo, S. A. Dayeh, and A. V. Davydov, Nat. Nanotechnol., 7, 749 (2012).

33. B. Key, R. Bhattacharyya, M. Morcrette, V. Seznéc, J.-M. Tarascon, and C. P. Grey, J. Am. Chem. Soc., 131, 9239 (2009).

34. T. K. Bhandakkar and H. T. Johnson, J. Mech. Phys. Solids, 60, 1103 (2012).

35. X. Zhang, W. Shyy, and A. M. Sastry, J. Electrochem. Soc., 154, A910 (2007).

36. J. C.-M. Li, Metall. Trans. A, 9, 1353 (1978).

37. M. J. Chon, V. A. Sethuraman, A. McCormick, V. Srinivasan, and P. R. Guduru, Phys. Rev. Lett., 107, 045503 (2011).

38. R. Fu, M. Xiao, and S. Y. Choe, J. Power Sources, 224, 211 (2013).

39. V. A. Sethuraman, M. J. Chon, M. Shimshak, V. Srinivasan, and P. R. Guduru, J. Power Sources, 195, 5062 (2010).

40. X. Zhang, A. M. Sastry, and W. Shyy, J. Electrochem. Soc., 155, A542 (2008).

41. H. Wu, G. Chan, J. W. Choi, Y. Yao, M. T. McDowell, S. W. Lee, A. Jackson, Y. Yang, L. Hu, and Y. Cui, Nat. Nanotechnol., 7, 310 (2012).

42. H. Guo, H. Zhao, C. Yin, and W. Qiu, Mater. Sci. Eng. B, 131, 173 (2006). 